\title{
The role of magnetic fields in the scattering of $p$-modes
}

\author{
A. Gascoyne and R. Jain
}

\author{
Applied Mathematics Department, The University of Sheffield, S3 7RH, UK \\ e-mail: [app07adg;R.Jain]@sheffield.ac.uk
}

Received 23 January 2009 / Accepted 13 May 2009

\begin{abstract}
Aims. We determine the direct and indirect effects of magnetic field on p-mode scattering.

Methods. We solve a set of magnetohydrodynamic equations using the Born approximation to determine phase shifts in p-modes due to a region of inhomogeneity. The region of inhomogeneity is a magnetic flux tube with the characteristics of flaring field lines. This enables us to investigate the magnetic field effects on the phase shifts.

Results. The magnetic configuration of our flux tube model plays a vital role in the phase shifts of p-modes. The suppression of sound speed and pressure within the flux tube region is not the only factor to consider in the scattering of $\mathrm{p}$-modes. There is a direct effect of the magnetic fields caused by the flaring of field lines on phase shifts.
\end{abstract}

Key words. sun: magnetic fields - sun: helioseismology - sun: oscillations - magnetohydrodynamics (MHD) - scattering

\section{Introduction}

Propagating modes can get scattered when travelling through an inhomogeneity and the resulting scattered modes carry information about this inhomogeneity. Scattering of $\mathrm{p}$-modes by sunspots have been studied, using various theoretical and observational helioseismic techniques. Braun et al. (1988) found that sunspots can absorb about half of incident p-mode power. Later Braun et al. (1992) observed, for the first time, phase shifts between the incident and outgoing p-modes with the same temporal frequency and radial orders. Further observations of p-mode phase shifts by sunspots were found by Braun (1995), paving the way for great insight into the subsurface structure of sunspots.

To understand and study how p-modes interact and consequently are scattered by inhomogeneities, various observational techniques have been put forward. Braun et al. (1988) (see also, Braun et al. 1992; Braun 1995) used a Fourier-Hankel decomposition method whereby the wave field is decomposed into its ingoing and outgoing components distinguished by Hankel functions. Another technique to observe the scattering of p-modes are investigated in Duvall et al. (1993). Here they extract timedistance information from temporal cross-correlations of the intensity fluctuations at subsurface inhomogeneities near sunspots. Similar methods and observational results can also be found in Kosovichev (1996), Kosovichev et al. (2000) and Couvidat et al. (2006).

Theoretical models of these inhomogeneities have been constructed to interpret the observations in an attempt to fully understand the structure of sunspots. Just like observations there are various techniques and approaches to model sunspots.

Rosenthal (1995) introduced the Born approximation to model p-mode scattering by a magnetic flux tube. This paper outlined the formulation of applying the Born approximation to calculate p-mode frequency shifts due to the presence of a fibril field. Fan et al. (1995) used a phenomenological model to calculate phase shifts. They modelled scattering by local inhomogeneities in wave speed, pressure and density consistent with those observed in active regions such as sunspots. They applied the Born approximation in the calculation of phase shifts, thus restricting the wavelength of the modes. Their approach gave good agreement with the observational data of Braun (1995).

Gordovskyy et al. (2006) and Gordovskyy \& Jain (2007) studied the scattering of acoustic waves by a magnetic flux tube embedded in a stratified polytropic atmosphere, taking into account magnetic field perturbations. Unlike Fan et al. (1995), Gordovskyy \& Jain (2007) included the Lorentz force in the governing equations. They also investigated the effect that flaring of fieldlines (observed in sunspot regions) has on phase shifts, by considering converging fieldlines. Similar to Fan et al. (1995), they applied the Born approximation to the wave equation with the added magnetic terms and calculated the phase shifts as a function of mode parameters. They found that for a strongly converging field the phase shift is noticeably increased compared to a weakly converging field, thus showing that sunspot magnetic fields play an important role in the scattering of p-modes. However, Gordovskyy \& Jain (2007) did not explicitly study the direct and indirect effects of magnetic fields on the phase shifts. It is not obvious from Gordovskyy \& Jain (2007) whether the p-mode phase shifts are a direct result of the magnetic fields or due to the thermal modification of the atmosphere in the presence of magnetic fields.

In this paper we use the magnetic flux tube model of Gordovskyy \& Jain (2007) to further understand how parameters in the source term influence the overall phase shifts. We also use the method introduced by Fan et al. (1995) and Rosenthal (1995) to set up our wave field taking into account magnetic field terms as done in Gordovskyy \& Jain (2007). We then investigate the effect of the Lorentz force by comparing the phase shifts with and without this term in the governing wave equation. This gives us insight into whether the scattering of $\mathrm{p}$-modes is affected predominantly by the suppression of pressure in the 
scattering region, or whether the presence of a magnetic field has an added effect due to magnetic pressure and tension. This is clear by the direct comparison of phase shifts for the two cases. In Sect. 2 we describe the construction of our magnetic flux tube model and its features. Section 3 describes the wave field and the use of the Born approximation to calculate phase shifts. Then in Sect. 4 we present our results and discuss our findings. Our main conclusions are mentioned in Sect. 5.

\section{The model}

Consider a vertical magnetic flux tube embedded in an adiabatically stratified polytropic atmosphere, which is axisymmetric. This causes local inhomogeneities in pressure, density and sound speed due to the presence of the tube. Thus, the equilibrium pressure $p$, density $\rho$ and sound speed $c^{2}$ consist of their background values denoted by the index "a", and their deviation, due to magnetic field $\boldsymbol{B}$, by the index "m". Thus, we have:

$$
\begin{aligned}
p & =p_{\mathrm{a}}(z)+p_{\mathrm{m}}(r, z), \\
\rho & =\rho_{\mathrm{a}}(z)+\rho_{\mathrm{m}}(r, z), \\
c^{2} & =c_{\mathrm{a}}^{2}(z)+c_{\mathrm{m}}^{2}(r, z) .
\end{aligned}
$$

We now make the following assumptions for the ambient pressure:

$p_{\mathrm{a}}(z)=p_{0}\left(1+\frac{z}{L}\right)^{\alpha+1}$,

where $z$ increases with depth. Thus, the ambient pressure increases with depth. Here $\alpha$ is the polytropic index given by the ratio of specific heats i.e. $\gamma=\frac{(\alpha+1)}{\alpha}$ which we set to $\alpha=2.5 ; L$ is the characteristic length scale which we set as $L=0.5 \mathrm{Mm}$ and $p_{0}=9 \times 10^{3} \mathrm{~Pa}$ is a constant for pressure at $z=0$ (pressure at the photosphere). Using the equation of motion in the absence of a magnetic field, we get

$\rho_{\mathrm{a}}(z)=\frac{1}{g} \frac{\mathrm{d} p_{\mathrm{a}}(z)}{\mathrm{d} z}=(\alpha+1) \frac{p_{0}}{g L}\left(1+\frac{z}{L}\right)^{\alpha}$,

where we assume constant gravity $g=2.7 \times 10^{2} \mathrm{~ms}^{-2}$ in $z$-direction. Thus, the ambient sound speed is

$c_{\mathrm{a}}^{2}=\gamma \frac{p_{\mathrm{a}}}{\rho_{\mathrm{a}}}$.

Now we define the magnetic profile first introduced by Gordovskyy \& Jain (2007)

$$
\begin{aligned}
& B_{z}(r, z)=B_{0}\left(1+\frac{z}{D}\right) \mathrm{e}^{-\frac{r^{2}}{R^{2}}\left(1+\frac{z}{D}\right)}, \\
& B_{r}(r, z)=-\frac{1}{2} B_{0} \frac{r}{D} \mathrm{e}^{-\frac{r^{2}}{R^{2}}\left(1+\frac{z}{D}\right)} .
\end{aligned}
$$

Here, $D$ is the convergence length, which corresponds to the depth at which the flux tube cross section decreases by a factor of $2 . R$ is the flux tube radius and $B_{0}$ is the characteristic magnetic field strength. We shall use the term strong (weak) convergence when the ratio $D / R<1(\geq 1)$. This magnetic profile is non-uniform in that the field lines tend to bend from a vertical position as $D$ is decreased, which results in a substantial radial component as $z \rightarrow 0$ and $r \rightarrow R$ (see Fig. 1). This represents the flaring of field lines observed in sunspots. Although we denote $R$ as the radius of the tube, there is a smooth transition from inside to outside of the tube due to the exponential component tending

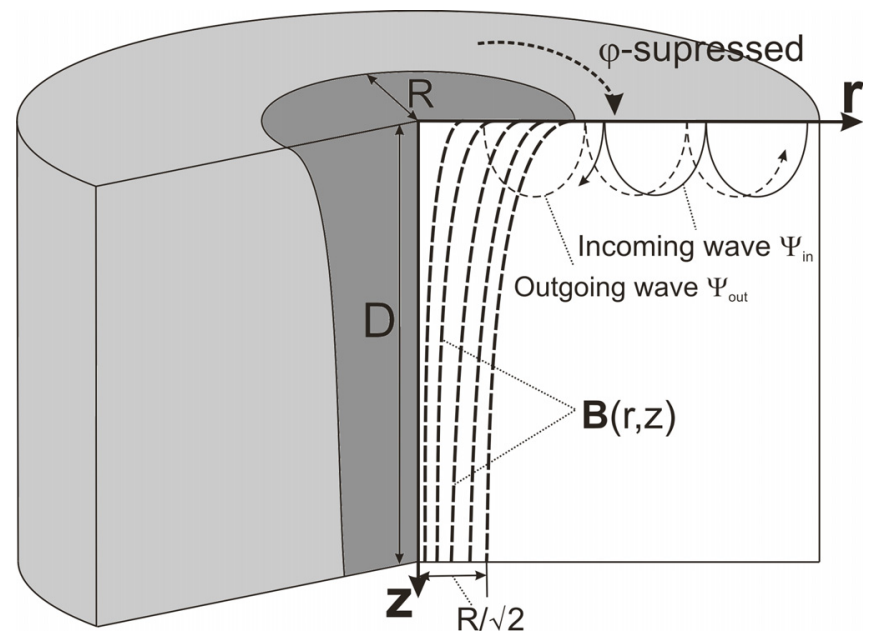

Fig. 1. The Model configuration. Dashed lines indicate magnetic fields and the wavefield consists of incoming $\Psi_{\text {in }}$ and outgoing $\Psi_{\text {out }}$ components.

to zero very quickly for $r>R$. Thus, there is no rigid boundary at the transition from magnetic to ambient (see also Jain \& Gordovskyy 2008).

Using the equation of motion the equilibrium pressure, density and sound speed due to the presence of magnetic field are given by

$$
\begin{aligned}
p_{\mathrm{m}}(r, z)= & -\frac{B_{0}^{2}}{2 \mu}\left[(1+z / D)^{2}+\frac{R^{2}}{8 D^{2}(1+z / D)}+\frac{r^{2}}{4 D^{2}}\right] \\
& \times \mathrm{e}^{-2 \frac{r^{2}}{R^{2}}\left(1+\frac{z}{D}\right)},
\end{aligned}
$$

$$
\begin{aligned}
\rho_{\mathrm{m}}(r, z)= & \frac{B_{0}^{2}}{g \mu}\left[\frac{r^{2}}{8 D^{3}(1+z / D)}-\frac{1}{D}(1+z / D)\right. \\
& \left.+\frac{R^{2}}{16 D^{3}}(1+z / D)^{2}\right] \mathrm{e}^{-2 \frac{r^{2}}{R^{2}}\left(1+\frac{z}{D}\right)},
\end{aligned}
$$

and

$c_{\mathrm{m}}^{2}(r, z)=\gamma\left(\frac{p_{\mathrm{a}}(z)+p_{\mathrm{m}}(r, z)}{\rho_{\mathrm{a}}(z)+\rho_{\mathrm{m}}(r, z)}-\frac{p_{\mathrm{a}}(z)}{\rho_{\mathrm{a}}(z)}\right)$.

In Fig. 2 we plot the sound speed distribution $c^{2}(r, z)$ normalised by the ambient sound speed $c_{\mathrm{a}}^{2}(z)$ showing the effect of convergence and field strength on the medium. We can see that the sound speed is suppressed in the flux tube region i.e., $R \leq 4 \mathrm{Mm}$, this suppression increases with magnetic field strength and with convergence strength. We have plotted contour lines to show where the sound speed approaches the value of the undisturbed atmosphere.

\section{Wavefield description}

In order to investigate the scattering of p-modes in and around the magnetic flux tube model we set up a wavefield consistent to that of our model i.e., cylindrical symmetry. Outside the flux tube region the wavefield consists of two components: incoming wavefield $\Psi_{\text {in }}$ and outgoing scattered waves represented by $\Psi_{\text {out }}$ (see Fig. 1). 

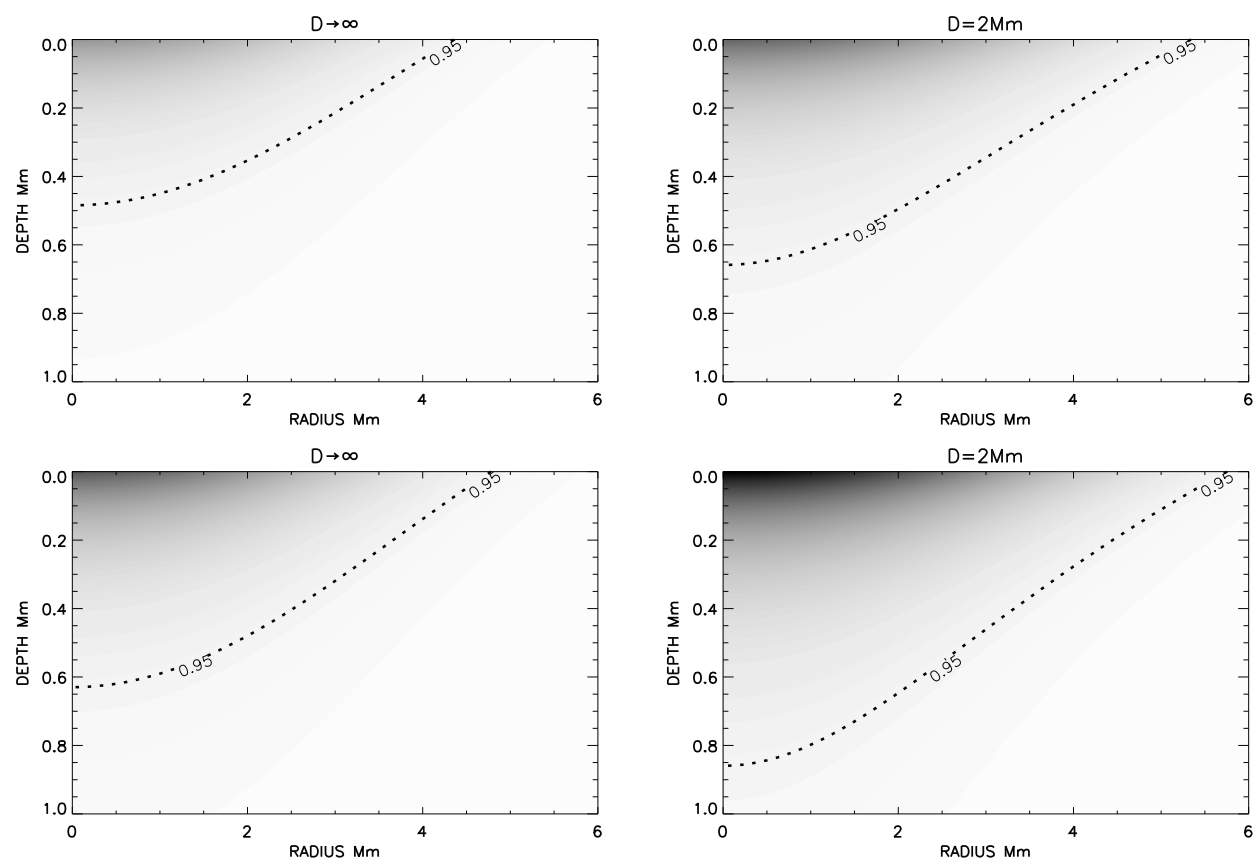

Fig. 2. Sound speed distribution $c^{2}(r, z)$ normalised by ambient sound speed $c_{\mathrm{a}}^{2}(z)$, for $R=4 \mathrm{Mm}$. Left panels are for $D \rightarrow \infty$ (i.e. no convergence) and right panels are for $D=2 \mathrm{Mm}$. The top and bottom panels are for $B_{0}=1.1 \mathrm{kG}$ and $1.4 \mathrm{kG}$ respectively. Dark areas represent a decrease in sound speed.

We consider the ideal MHD equations for a gravitationally stratified polytropic atmosphere. Thus, we have the following linearised MHD equations for wave perturbations $p^{\prime}, \rho^{\prime}$ and $\boldsymbol{v}^{\prime}$ :

$\frac{\partial \rho^{\prime}}{\partial t}+\nabla \cdot\left(\rho \boldsymbol{v}^{\prime}\right)=0$

$$
\begin{aligned}
\rho \frac{\partial \boldsymbol{v}^{\prime}}{\partial t}+\nabla p^{\prime}- & \frac{1}{\mu}\left[(\boldsymbol{\nabla} \times \boldsymbol{B}) \times \boldsymbol{B}^{\prime}\right] \\
& -\frac{1}{\mu}\left[\left(\boldsymbol{\nabla} \times \boldsymbol{B}^{\prime}\right) \times \boldsymbol{B}\right]-\rho^{\prime} \boldsymbol{g}=0,
\end{aligned}
$$

$\left(\frac{\partial p^{\prime}}{\partial t}+\left(\boldsymbol{v}^{\prime} \cdot \boldsymbol{\nabla}\right) p\right)-c^{2}\left(\frac{\partial \rho^{\prime}}{\partial t}+\left(\boldsymbol{v}^{\prime} \cdot \boldsymbol{\nabla}\right) \rho\right)=0$

$\frac{\partial \boldsymbol{B}^{\prime}}{\partial t}-\boldsymbol{\nabla} \times\left(\boldsymbol{v}^{\prime} \times \boldsymbol{B}\right)=0$.

We now take the time dependence of $p^{\prime}, \rho^{\prime}$ and $\boldsymbol{v}^{\prime}$ as $e^{i \omega t}$ and eliminate $\rho^{\prime}$ and $\boldsymbol{v}^{\prime}$ from Eqs. (12) to (15). After rearranging the terms we have the inhomogeneous wave equation for pressure perturbations (see also Fan et al. 1995)

$\mathcal{L} p^{\prime}+\frac{\omega^{2}}{(z+L)^{\alpha+1}} p^{\prime}=\mathcal{S}$,

where,

$$
\begin{aligned}
& \mathcal{L}=\frac{g}{\alpha(z+L)^{\alpha}}\left[\nabla^{2}-\frac{\alpha}{(z+L)} \frac{\partial}{\partial z}+\frac{\alpha}{(z+L)^{2}}\right], \\
& \mathcal{S}=\frac{g}{\alpha(z+L)^{\alpha}}\left[i \omega \mathcal{F}+\frac{g}{i \omega} \frac{\partial \mathcal{F}}{\partial z}+\frac{1}{i \omega} \boldsymbol{\nabla} \cdot \mathcal{U}\right], \\
& \mathcal{F}=\frac{1}{c_{\mathrm{a}}^{2}}\left(\boldsymbol{v}^{\prime} \cdot \boldsymbol{\nabla}\right) p_{\mathrm{m}}-\left(\boldsymbol{v}^{\prime} \cdot \boldsymbol{\nabla}\right) \rho_{\mathrm{m}}+\rho_{\mathrm{a}} \frac{c_{\mathrm{m}}^{2}}{c_{\mathrm{a}}^{2}}\left(\boldsymbol{\nabla} \cdot \boldsymbol{v}^{\prime}\right),
\end{aligned}
$$

$$
\begin{aligned}
\boldsymbol{U}= & \frac{1}{\mu}\left[(\boldsymbol{\nabla} \times \boldsymbol{B}) \times\left\{\boldsymbol{\nabla} \times\left(\boldsymbol{v}^{\prime} \times \boldsymbol{B}\right)\right\}\right] \\
& -\frac{1}{\mu}\left[\left\{\boldsymbol{\nabla} \times \boldsymbol{\nabla} \times\left(\boldsymbol{v}^{\prime} \times \boldsymbol{B}\right)\right\} \times \boldsymbol{B}\right] .
\end{aligned}
$$

Here $\boldsymbol{s} \mathcal{U}$ is the term which describes the effect of the Lorentz force which is omitted in Fan et al. (1995).

To solve Eq. (16) we use the Born approximation (Fan et al. 1995), thus we set $\mathcal{S}=0$ and solve the homogeneous wave equation, thus acquiring $p_{(0)}^{\prime}$. We then construct the approximate source term,

$\mathcal{S}_{(0)}=\frac{g}{\alpha(z+L)^{\alpha}}\left[i \omega \mathcal{F}_{(0)}+\frac{g}{i \omega} \frac{\partial \mathcal{F}_{(0)}}{\partial z}+\frac{1}{i \omega} \boldsymbol{\nabla} \cdot \boldsymbol{\mathcal { U }}_{(0)}\right]$.

The solutions to the homogeneous wave equation, for azimuthal order $m=0$, under the physical boundary conditions form a set of orthogonal eigenfunctions (Fan et al. 1995),

$\psi_{n, k}(r, z)=\boldsymbol{\Phi}_{n, k}(z) J_{0}(k r)$

where

$\omega_{n, k}^{2}=g k\left(\frac{\alpha+2 n}{\alpha}\right)$

corresponds to the eigenfrequencies of the different modes of oscillation, $n$, and $k$ is the horizontal wavenumber. The radial component of the eigenfunctions are represented by the Bessel function $J_{0}(k r)$. The horizontal component is defined as,

$$
\begin{aligned}
\boldsymbol{\Phi}_{n, k}(z)= & \sqrt{\frac{\Gamma(\alpha+n)}{n !}} \frac{1}{\Gamma(\alpha)} \mathrm{e}^{-k(z+L)}(2 k)^{\frac{\alpha}{2}}(z+L)^{\alpha} \\
& \times \mathcal{M}(-n, \alpha, 2 k(z+L))
\end{aligned}
$$

which contains the Kummer function $\mathcal{M}(-n, \alpha, 2 k(z+L))$.

To calculate the phase shifts of propagating waves caused by the proposed magnetic flux tube we use the method shown in 

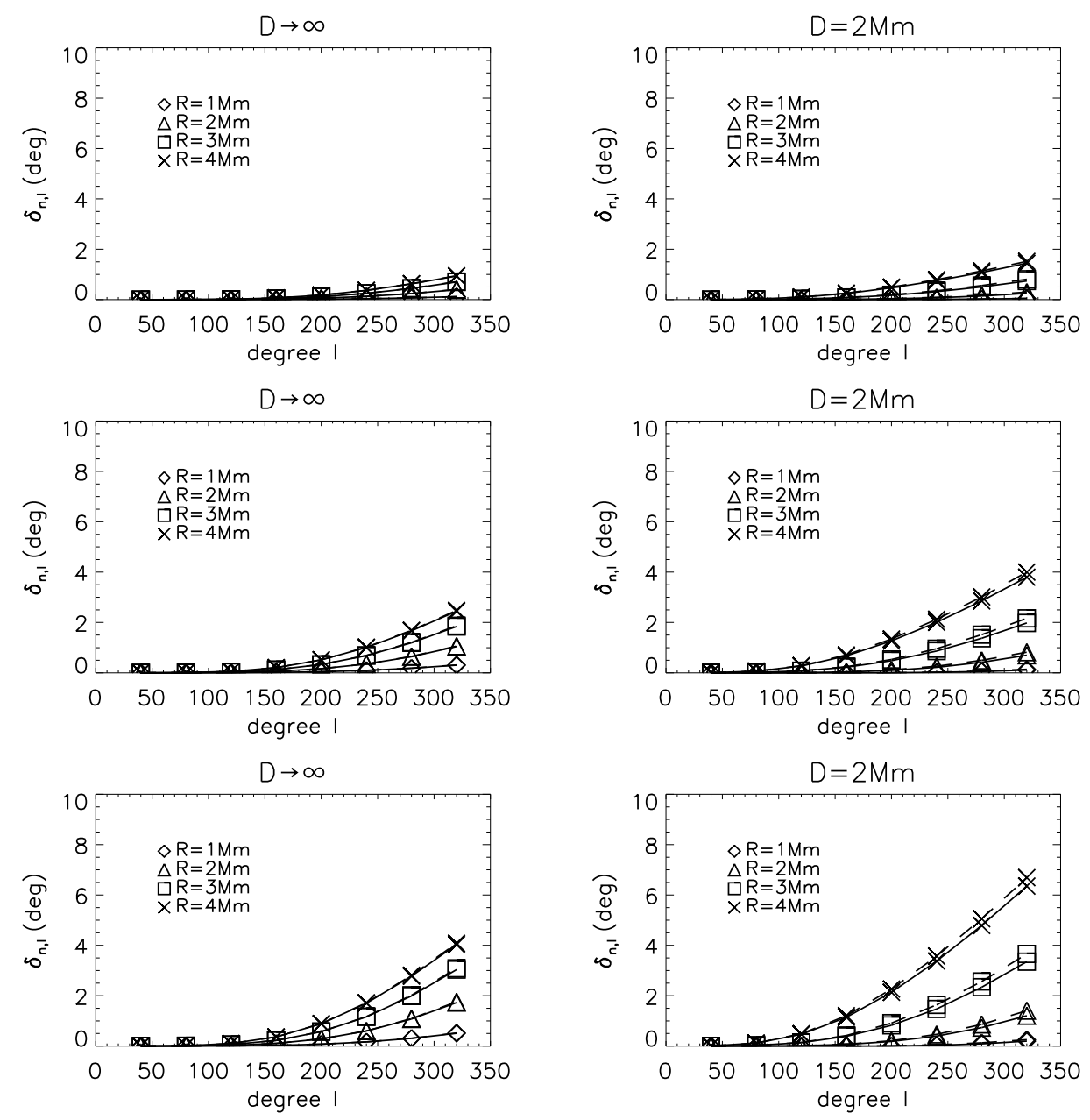

Fig. 3. Phase shifts $\delta_{n, l}$ as a function of angular degree $l$ for $n=1$ and various flux tube radii. Left panels are for $D \rightarrow \infty$ (i.e. no convergence) and right panels are for $D=2 \mathrm{Mm}$. The top, middle and bottom panels are for $B_{0}=0.7,1.1$ and $1.4 \mathrm{kG}$ respectively. The dashed lines indicate the phase shifts in the absence of the $\mathcal{U}$ term given by Eq. (20).

Fan et al. (1995) and Gordovskyy \& Jain (2007), thus we compute the following integral for a truncated polytrope (Hindman \& Jain 2008):

$\tan \delta_{n, l}=\frac{2 \pi}{\omega^{2}} \int_{0}^{\infty} \mathrm{d} z \int_{0}^{\infty} r \mathrm{~d} r\left[k^{2} \boldsymbol{\psi}_{n, k}(r, z) \mathcal{S}_{(0)}\right]$.

We calculate this integral numerically for various model parameters ensuring that the Born approximation is valid, i.e., the wavelength of the propagating wave modes are greater than the radius of the scattering region. We must also restrict the magnetic field strength to $B_{0} \leq 1.4 \mathrm{kG}$ as the Born approximation is valid only for small source term $\boldsymbol{S}_{(0)}$. Also for stronger magnetic field strengths and convergence, pressure inside the tube becomes negative.

\section{Results and discussion}

In this section we discuss the phase shifts calculated numerically using Eq. (25). The phase shifts are plotted as a function of angular degrees $l ; n$ is the radial order which we take as 1 or 2 . We want to study the effect that different magnetic flux tube parameters have on the phase shifts of p-modes. Thus, we plot phase shifts as a function of degree for different flux tube radii and field strengths. We also show the effect of magnetic field term on the phase shifts for various model configurations by including and omitting the $\mathcal{U}$ term in the source term. We further investigate whether the magnetic field has a direct or an indirect effect on acoustic wave scattering by fixing $D / R$ and comparing the change in phase shifts for different $R$. The following subsections discuss each of these effects.

\subsection{Effect of magnetic flux tube radius, angular degree, radial order and field strength}

By analysing plots in Figs. 3 and 4 we can clearly see that increasing the radius of the flux tube increases the phase shifts of the p-modes for all wave parameters. This is expected because as we increase the radius $R$, the region of suppressed sound-speed becomes larger; thus a larger inhomogeneity. We do not consider very large radii as the Born approximation forces limitations on the radius of the scattering region and the wavelength of modes. We have ensured that either $k R<1$ or $k R \approx 1$ (Birch et al. 2001).

The phase shift functions $\delta_{n, l}$ versus angular degree $l$ describe a behaviour similar to that found in Fig. 8 of Fan et al. (1995). As $l$ increases the phase shift increases, but this increase is less dramatic when $l$ becomes greater than 200 .

We plot the phase shifts for two different radial orders $n=1$ and $n=2$ shown in Figs. 3 and 4. In agreement with Fan et al. (1995) we find that phase shifts for the fundamental mode, $n=0$, 

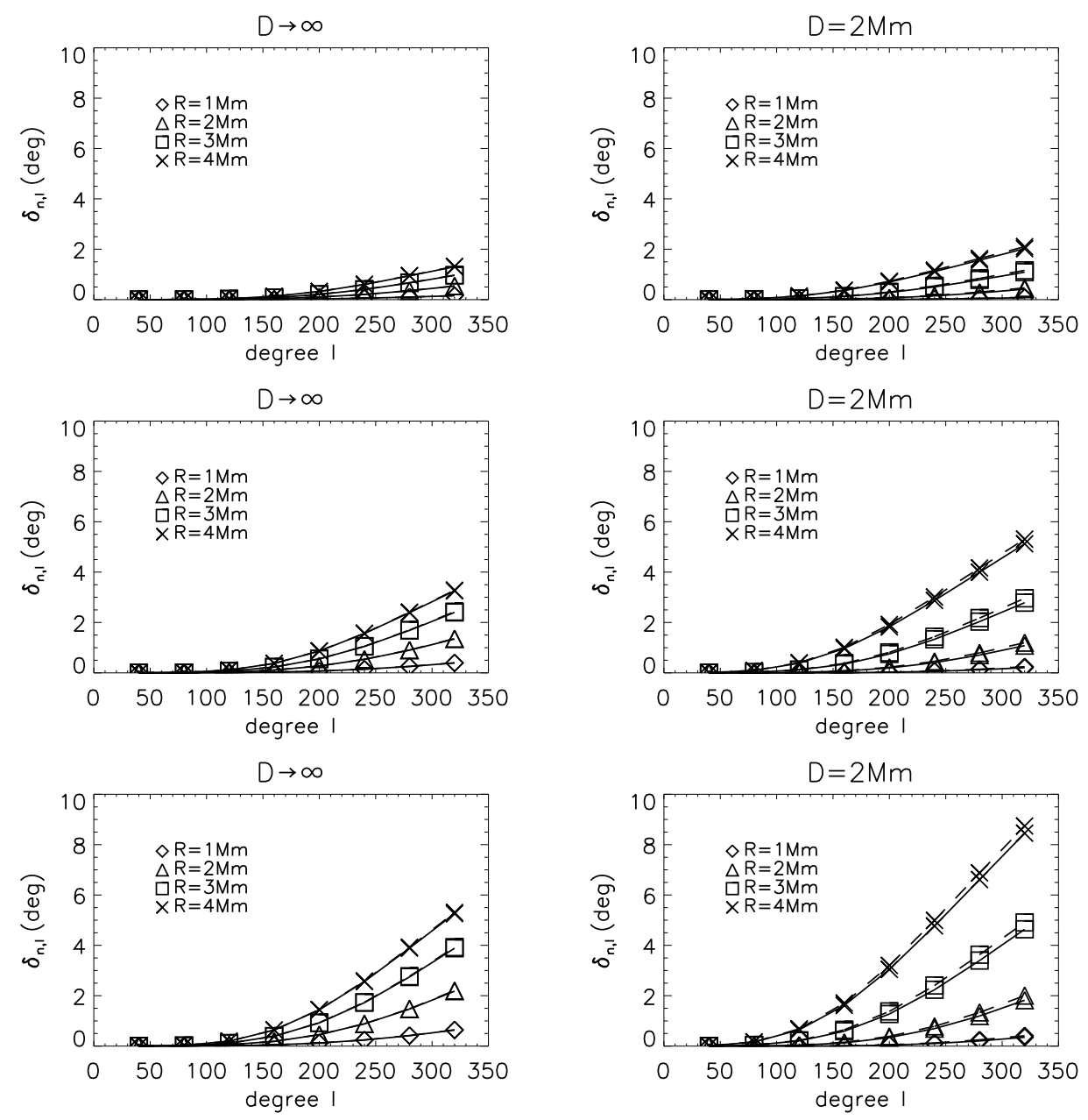

Fig. 4. Phase shifts $\delta_{n, l}$ as a function of angular degree $l$ for $n=2$ and various flux tube radii $R$. Left panels are for $D \rightarrow \infty$ (i.e. no convergence) and right panels are for $D=2 \mathrm{Mm}$. The top, middle and bottom panels are for $B_{0}=0.7,1.1$ and $1.4 \mathrm{kG}$ respectively. The dashed lines indicate phase shifts in the absence of the $\mathcal{U}$ term given by Eq. (20).

are zero and that the phase shifts increase with radial order (at least up to $n=2$ ).

We expect the magnetic field strength to have a strong impact on phase shifts (see e.g. Braun 1995) so we plot $\delta_{n, l}$ for three different field strengths $B_{0}=0.7,1.1$ and $1.4 \mathrm{kG}$ shown in Figs. 3 and 4. As predicted, they show an increase in phase shift as we increase $B_{0}$, due to the increased suppression of sound speed for higher $B_{0}$. As mentioned earlier there is an upper bound for $B_{0}$ which we take as $1.4 \mathrm{kG}$, an increase in this value would result in negative pressure inside the tube which is obviously meaningless.

\subsection{Effect of converging magnetic fieldlines}

Convergence of the magnetic fieldlines can be separated into two categories: strong convergence $D<R$ and weak convergence $D \geq R$. We have plotted the two extremes of convergence, $D \rightarrow \infty$ and $D=2 \mathrm{Mm}$. It can be seen from Figs. 3 and 4 that phase shifts significantly increase with convergence. This indicates that substantial flaring of field lines has a greater effect on the suppression of sound speed within a magnetic flux tube which then results in greater phase shifts. This increase is more substantial for angular degree $l \geq 100$ and for stronger magnetic fields. This is in agreement with the findings of Gordovskyy \& Jain (2007).
Note that when studying the sound speed profiles of the flux tube region it is hard to distinguish the difference between a converging $\left(D=2 \mathrm{Mm}, B_{0}=1.1 \mathrm{kG}\right)$ and non-converging $(D \rightarrow \infty$, $B_{0}=1.4 \mathrm{kG}$ ) magnetic flux tube, as shown in Fig. 2. This means that it would be difficult to make inferences on magnetic stucture when inverting the observational data to get sound speed profiles.

\subsection{Direct effect of the magnetic field on phase shifts}

In Figs. 3 and 4 we plot phase shifts with and without the term $\mathcal{U}$ in the source term $\mathcal{S}$ (see Eq. (20)). These are represented by solid and dashed lines respectively. This is done in order to investigate the direct effect of magnetic field on phase shifts. It is clear from the figures that for weak convergence $(D \geq R)$, there is no significant difference between the two cases but for strong convergence $(D<R)$, the phase shifts are slightly reduced when the magnetic field term $\mathcal{U}$ is included. This reduction in the phase shift due to direct magnetic field effect will be more pronounced for stronger field $\left(B_{0}>1.5 \mathrm{kG}\right)$ though this is not shown here due to the restrictions imposed by the Born approximation.

In order to investigate in details, the dependence on the magnetic fields the phase shifts are calculated and plotted in Fig. 5 as a function of magnetic field strengths for various flux tube 

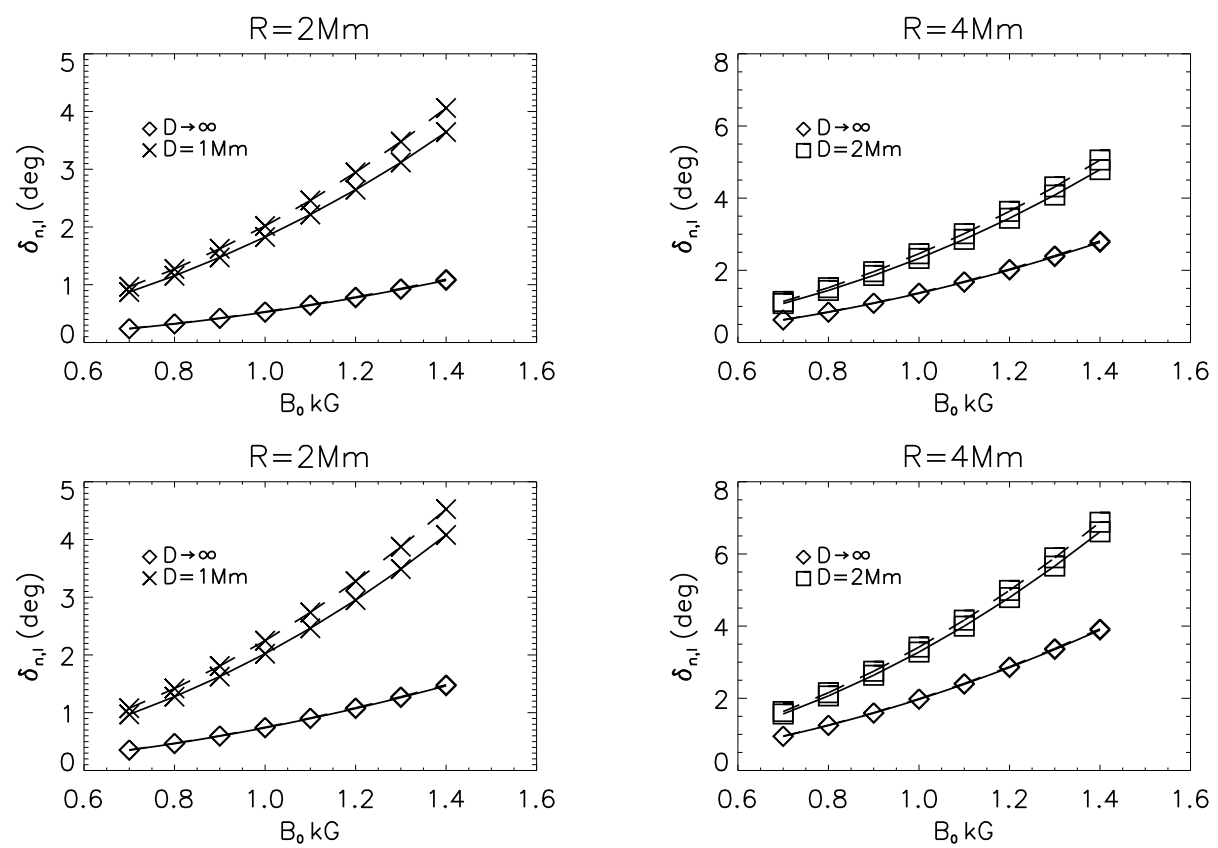

Fig. 5. Phase shifts $\delta_{n, l}$ as a function of magnetic field strength $B_{0} \mathrm{kG}$ for $l=280$ and different convergence lengths $D$ Mm. Left and right panels correspond to radius $R=2$ and $4 \mathrm{Mm}$ respectively. Top and bottom panels are for $n=1$ and 2 respectively. The dashed lines indicate the phase shifts in the absence of the $\mathcal{U}$ term given by Eq. (20).
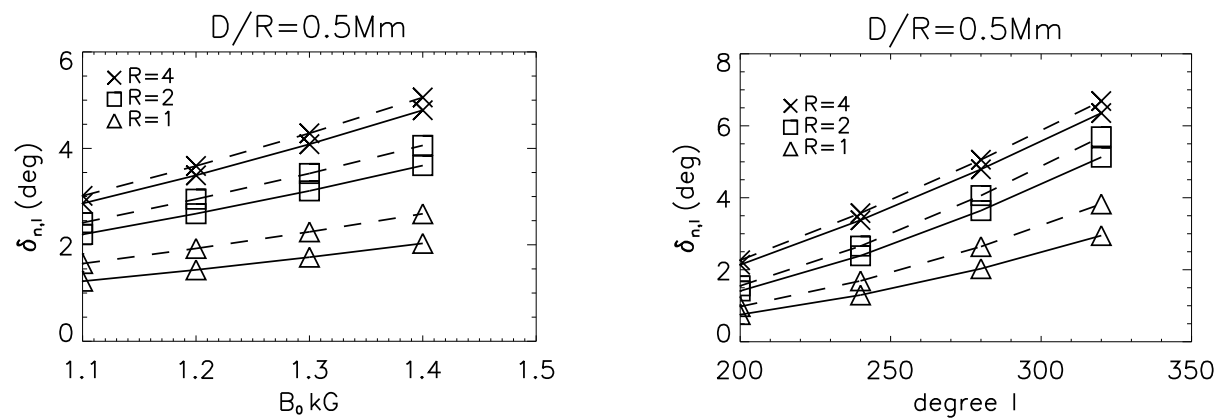

Fig. 6. Phase shifts $\delta_{n, l}$ as a function of magnetic field strength $B_{0} \mathrm{kG}$ (left panel) for $l=280$ and a function of angular degree $l$ (right panel) for $B_{0}=1.4 \mathrm{kG}$. We have set $n=1$ and the ratio $D / R=0.5$ for various $R$. The dashed lines indicate the phase shifts in the absence of the $\mathcal{U}$ term given by Eq. (20).

parameters. Note that as $D \rightarrow \infty$, the solid and dashed lines overlap. This is due to the fact that for $D \rightarrow \infty$ (non-converging field case), there is no magnetic tension or radial component of magnetic pressure, as a result of which there is no contribution of $\mathcal{U}$ term in the phase shifts.

As we decrease the convergence length, the magnitude of the phase shifts increases which is expected as there is more tension in the fieldlines. The phase shifts also increase with the magnetic field strength $B_{0}$ because of more suppression in sound speed; this is also true when we increase radius $R$ and radial order $n$.

To further understand the direct and indirect effects of magnetic fields on phase shifts and the role of the term $\mathcal{U}$ (see Eq. (20)), we plot in Fig. 6 the phase shifts for fixed $D / R=0.5$ verses $B_{0}$ (left panel) and angular degree $l$ (right panel). The dashed (solid) lines indicate phase shifts due to the absence (presence) of the $\mathcal{U}$ term. As we decrease $R$ (consequentially decreasing $D$ ) the difference between dashed and solid lines increases. This is due to the fact that although the magnetic tension is the same for all three cases, magnetic pressure is less for smaller R. Also, the size of the equipartition layer is small for smaller $R$ resulting in less efficient mode conversion
(Cally \& Bogdan 1993). This is illustrated clearly in Fig. 7 where we have shown the equipartition layer for each of the cases investigated in Fig. 6. It can be seen from Figs. 6 and 7 that in the current model, the direct effect of magnetic field (magnetic tension, magnetic pressure and mode conversion) is to further reduce the phase shifts. This phase shift difference also increases with magnetic field strength and angular degree $l$.

\section{Conclusions}

In this paper, we have studied the phase shifts of p-modes by considering magnetic flux tube as a local inhomogeneity. We computed the phase shifts using the Born approximation and investigated the effect of different flux tube parameters on the scattering of p-modes. Our main conclusion is that the phase shift depends on the direct (e.g. magnetic tension, magnetic pressure and the process of mode-conversion) and indirect (e.g. modifications to the ambient pressure, density and sound speed) effects of magnetic fields. Although the indirect effects have a dominant contribution, one should not ignore direct effects of magnetic fields when using observational data of phase shifts 


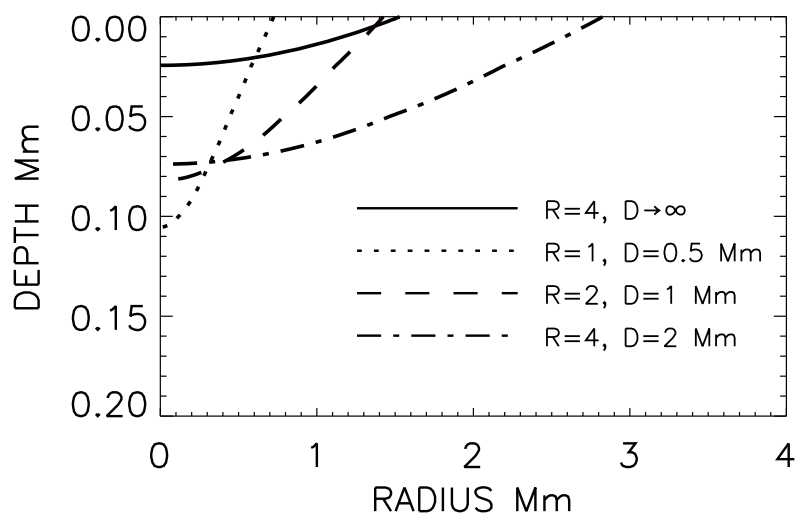

Fig. 7. Plot showing the equipartition layer $\left(v_{A}^{2}=c^{2}\right)$ for $B_{0}=1.4 \mathrm{kG}$. The solid line is for the vertical field case where as the dotted, dashed and the dot-dashed lines are all for $D / R=0.5$.

to make inferences on the structure of strong magnetic flux tube regions such as sunspots. In particular, strongly converging fields of high strength could have appreciable effect on the phase shifts of p-modes. Inversions of observed travel-times or phase shifts in and around a sunspot could easily give incorrect sound speed distribution if the geometry of the sunspot is not accurately known. By ignoring magnetic tension terms (for example, in penumbrae) during inversions one could easily underestimate the sound speed suppression compared to the surroundings or overestimate the magnetic field strength by presuming vertical field geometry.

It is important to note from the present study that the phase speeds obtained from the ray analysis does not necessarily directly correspond to the background sound speed, since the magnetic field is also playing a direct role in altering the phase. However, since the magnetic tension terms will not be the same for all sunspots and other magnetically active regions, we cannot suggest a unique way to invert the observational data for the sound speed distribution in these regions. More future work will be directed towards this aspect.

As clearly seen from the present work, the inclination of the field lines near the surface of a magnetic structure are affected by the convergence $D / R$, which in turn influences the magnitude of the phase shifts. Although this ratio cannot be currently measured accurately from observations, one can constrain it for this model by surface observations of the vector magnetic field of sunspots (see e.g. Schunker et al. 2008). Thus we expect typical value of $D / R$ to be $0.4<D / R<2$ for the inclination observed in the sunspots between umbrae and penumbrae.

In this paper we used the Born approximation to calculate phase shifts; this imposes restrictions on our model parameters. The inhomogeneity must not be too strong or else the approximation will not be accurate, so interpreting our results for any observed phase shifts higher than $60^{\circ}$ should be considered with caution. Our future work will address this issue. The calculations presented here are very sensitive to the physical boundary chosen at the surface but we want to emphasize the effect of $\mathcal{U}$ term (magnetic) instead of the absolute value of the phase shifts.

In the current model, it appears that if magnetic and thermal perturbations are to be considered separately then although they compete against each other, thermal perturbations dominate. In our future work we will investigate in detail the effects of mode conversion and reflection which as shown in Fig. 6, clearly play an important role. It has been known that such effects are significant for p-mode phase shifts. For example, Cally et al. (2003) investigated mode-conversion mechanism in inclined fields and reproduced the observed phase shifts calculated from Hankel analysis by Braun (1995). Recently, Lindsey \& Braun (2005) and Schunker et al. (2008) have shown that the phase shift of the outward propagating waves is opposite to the phase shift of the inward propagating waves in stronger, vertical fields. Couvidat \& Rajaguru (2007) also report possible contamination of helioseismic inversions for sound speed beneath sunspots, possibly due to near surface magnetic field effects. Obviously the choice of the surface magnetic field and the profile of the background atmosphere can vary results but it is becoming evident that in addition to purely thermal effects, the direct effects of magnetic field play a significant role in altering the phase shift of $\mathrm{p}$-modes.

Acknowledgements. We are thankful to M. Gordovskyy for many useful discussions. We also appreciate constructive comments from an anonymous referee which have helped to improve this manuscript. This work is supported by UK STFC studentship.

\section{References}

Birch, A. C., Kosovichev, A. G., Price, G. H., \& Schlottmann, R. B. 2001, ApJ, 561, L229

Braun, D. C. 1995, ApJ, 451, 859

Braun, D. C., Duvall, T. L., \& LaBonte, B. J. 1988, ApJ, 335, 1015

Braun, D. C., Duvall, T. L., LaBonte, B. J., et al. 1992, ApJ, 391, L113

Cally P. S., \& Bogdan, T. J. 1993, ApJ, 402, 732

Cally, P. S., Crouch, A. D., \& Braun, D. C. 2003, MNRAS, 346, 381

Couvidat, S., \& Rajaguru, S. P. 2007, ApJ, 661, 558

Couvidat, S., Birch, A. C., \& Kosovichev, A. G. 2006, ApJ, 640, 516

Duvall, T. L., Jefferies, S. M., Harvey, J. W., \& Pomerantz, M. A. 1993, Nature, 362,430

Fan, Y., Braun, D. C., \& Chou, D. Y. 1995, ApJ, 451, 877

Gordovskyy, M., \& Jain, R. 2007, ApJ, 661, 586

Gordovskyy, M., Jain, R., \& Thompson, M. J. 2006, Beyond the Spherical Sun: a new era of helio and astero-seismology, proceedings of SOHO18/GONG2006/HeLAS I, Sheffield, UK, ESA SP-624

Hindman, B. W., \& Jain, R. 2008, ApJ, 677, 769

Jain, R., \& Gordovskyy, G. 2008, Sol. Phys., 251, 361

Kosovichev, A. G. 1996, ApJ, 461, L55

Kosovichev, A. G., Duvall, T. L., \& Sherrer, P. H. 2000, Sol. Phys., 192, 159

Lindsey, C., \& Braun, D. C. 2005, ApJ, 620, 1118

Rosenthal, C. S. 1995, ApJ, 438, 434

Schunker, H., Braun, D. C., Lindsey, C., \& Cally, P. S. 2008, Sol. Phys., 251, 341 